\title{
Electronic Structure of Ferrocene-Substituted Cavitands: A QTAIM and NBO Study
}

\author{
Tímea R. Kégl, László Kollár, and Tamás Kégl \\ Department of Inorganic Chemistry and János Szentágothai Research Center, University of Pécs, \\ MTA-TKI Research Group for Selective Chemical Syntheses, Ifjúság Útja 6, Pécs 7624, Hungary
}

Correspondence should be addressed to Tamás Kégl; tkegl@gamma.ttk.pte.hu

Received 1 November 2013; Accepted 24 December 2013; Published 10 February 2014

Academic Editor: Anton Kokalj

Copyright (C) 2014 Tímea R. Kégl et al. This is an open access article distributed under the Creative Commons Attribution License, which permits unrestricted use, distribution, and reproduction in any medium, provided the original work is properly cited.

Ferrocene-substituted tetrakis(methyl)resorcin[4]arenes have been investigated by means of DFT calculations employing the gradient-corrected PBEPBE functional. In comparison with ferrocene and simple ansa-ferrocenes containing 2-4 bridging methylene groups, it was found that the tilt angle of the functionalized cyclopentadienyl (Cp) rings strongly influences the electron density distribution of the ferrocenyl moieties. According to NBO analyses, the iron atoms in the cavitands are more positive in comparison to those in ferrocene, whereas they are less positive in ansa-ferrocenes. The partial charges of carbon atoms belonging to $\mathrm{Cp}$ rings show some correlation with the tilt angle.

\section{Introduction}

Metallocenes are among the first known organometallic compounds. Since the discovery of ferrocene [1] and the elucidation of its bispentahapto sandwich structure, biscyclopentadienyl-transition metal complexes have been in the focus of many important developments in modern organometallic chemistry.

In biscyclopentadienyl-transition metal complexes, the cyclopentadienyl $(\mathrm{Cp})$ rings are relatively inert moieties in most reactions. The $\mathrm{Cp}$ rings can function as scaffolding of the upper and lower frames and define the reaction space of the attached metal. Moreover, the $\mathrm{Cp}$ ring can be varied in an almost unlimited number of ways in order to modify the steric properties of the complex and the electronic properties of the metal. A commonly used alteration to the metallocene ligand framework is the inclusion of a linking group between the two $\mathrm{Cp}$ rings called an interannular bridge. Complexes of this class were originally called metallocenophanes; however, the term "ansa-metallocene" is now more commonly used [2]. The Latin prefix ansa (meaning "handle") was introduced by Smith et al. [3], referring to the bent handle functional group connecting to the two Cp rings at both ends. Ansametallocenes became very popular in the polymer industry as, for example, an excellent catalyst in the synthesis of highly isotactic polypropylene [4].

The amount of distortion from the normal metallocene geometry that is caused by the bridging group $\mathrm{X}$ is depicted in Scheme 1. Several geometrical parameters can be defined describing the geometries of ansa-metallocenes [2], from which we will focus on two fundamental values within this study. The degree of ring tilt is represented by angle $\alpha$, whereas angle $\beta$ denotes the deviation of the $\mathrm{X}-\mathrm{C}_{\mathrm{ipso}}$ axis from the ring plane.

The reactivity of metallocenes can substantially be influenced by the bridge. Lentzner and Watts studied the effect of the bridge on the reactivity of ferrocenophanes [5]. The tetramethylene bridge enforces some bending of the $\mathrm{Cp}$ rings and enhances the Lewis basicity of the iron center in comparison to that in $\mathrm{Cp}_{2} \mathrm{Fe}$ but makes the aromatic rings resistant to Friedel-Crafts acylation and to lithiation by Wilkinson et al. [6].

The introduction of an ethylene bridge between Cp rings has a strong effect on the chromocene system [7]. Whereas the carbonyl complex $\mathrm{Cp}_{2} \mathrm{CrCO}$ cannot be isolated due to the reversible binding of $\mathrm{CO}$ by chromocene [8], various ansa-chromocenes were prepared and crystallographically characterized $[9,10]$. DFT calculations indicated that 

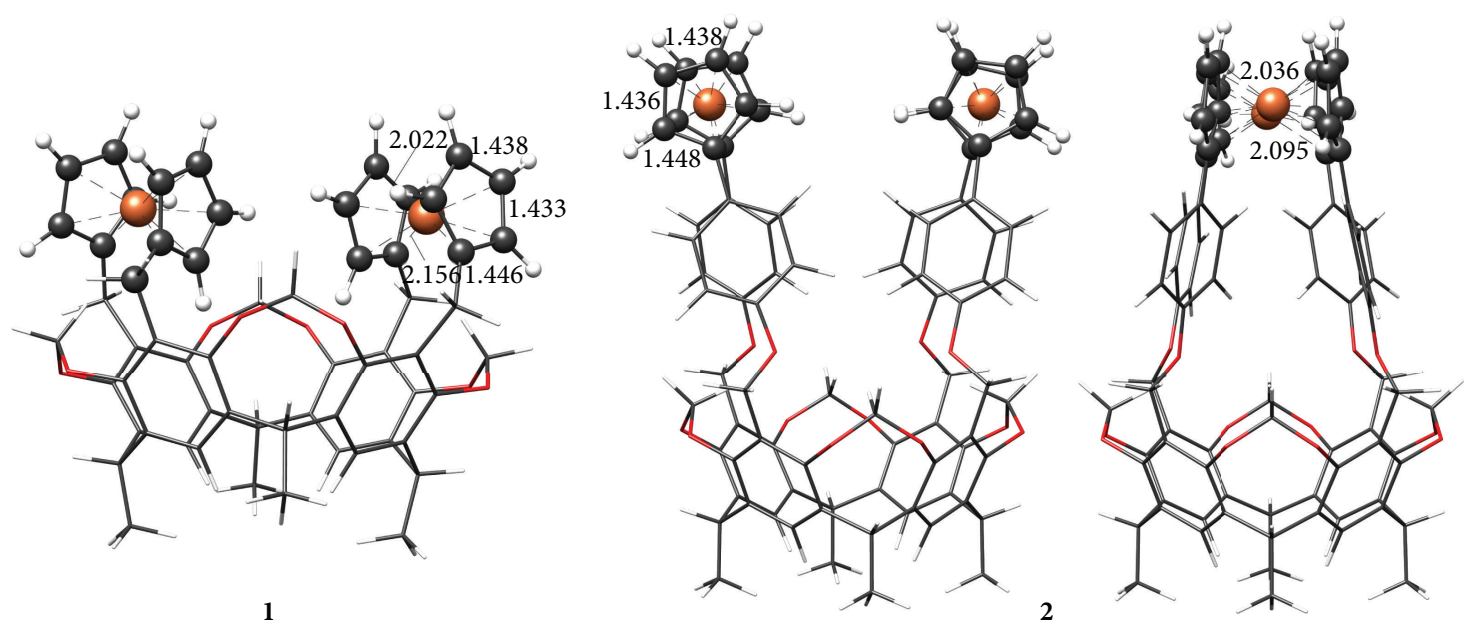

FIGURE 1: Computed structures of complexes 1 and $\mathbf{2}$ (from two viewpoints). Bond lengths are given in $\AA$.

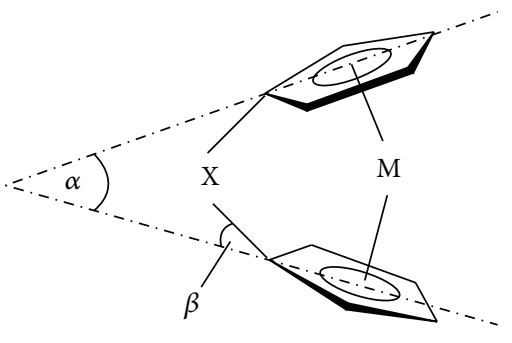

SCHEME 1

the stabilization of the CO complex of ansa-chromocene is the consequence of the stability of the triplet metallocene product. However, in the ansa-bridged free chromocene the rings are unable to relax to a near parallel structure, therefore the triplet state is of less advantage [11].

The resorcin[4]arenes and the related cavitands are macrocyclic compounds consisting of multiple aromatic rings connected by different linkers [12]. Cavitands are known to offer excellent points of departure for the construction of large bowl-shaped molecular entities possessing a well-formed hydrophobic cavity. Therefore, cavitands and similar structures show promise with potential applications as gas sensors, nanoreactors, and drug delivery systems. Cavitands have also found a wide application in the preparation of capsule-like self-assemblies. Ferrocene-substituted resorcinarenes were found to be redox-switchable dynamic systems, in which the ferrocene moieties serve as electroactive hydrophobic fragments [13]. Moreover, decamethylruthenocene was found to act as template for the self-assembly of a Cmethylcalix[4] resorcinarene/bipyridine/decamethylruthenocene supramolecular crystal [14].

The first goal of this study is to describe the geometry and electronic structure of tetrakis(methyl)resorcin[4] arene (1) and tetrakis(phenoxymethyl)resorcin[4]arene (2) containing ferrocene moieties. The ferrocenyl groups provide some prediction for their stability and applicability by the functionalization of cavitands with cyclopentadiene followed by deprotonation and metallocene formation by the addition of $\mathrm{Fe}(\mathrm{II})$ salts. The second purpose of this paper is to give some details for the electron density distribution of ferrocene-substituted cavitands in comparison to simple ansa-ferrocenes containing 2-4 bridging methylene groups, as model compounds.

\section{Computational Details}

For all the calculations the PBEPBE gradient-corrected functional by Perdew et al. [15] was selected using the Gaussian 09 suite of programs [16]. For iron, the triple-zeta basis set by Schaefer and coworkers was applied and denoted as TZVP [17], whereas the 6-31G(d,p) basis set [18] was employed for every other atom. Local minima were identified by the absence of the negative eigenvalues in the vibrational frequency analyses, whereas the Hessian matrix of transition states has only one negative eigenvalue. For the NBO calculations the GENNBO 5.0 program was utilized [19]. For the QTAIM studies the AIMAll software was employed [20].

\section{Results and Discussion}

The functionalization of resorcin[4] arenes with ferrocenes is predicted to result in well-organized structures with symmetries very close to $C_{2}$. The computed geometries of ferrocenesubstituted tetrakis(methyl)resorcin[4] arene (1) and tetrakis(phenoxymethyl)resorcin[4]arene (2) are depicted in Figure 1. For a better visibility, complex $\mathbf{2}$ is shown from two viewpoints emphasizing its peculiar structure arranged by the closure of Cp rings around the iron centers forming formally a dimer of ansa-ferrocenes.

For comparison, the geometries of ferrocene and simple ansa-ferrocenes, containing bridges formed from $2-4 \mathrm{CH}_{2}$ groups, have been computed as well and illustrated in Figure 2 . The unsubstituted ferrocene is denoted as $\mathbf{3}$, whereas ansaferrocenes with ethylene, 1,3-propylene, and 1,4-butylene bridges are denoted as $\mathbf{4 , 5}$, and $\mathbf{6}$, respectively. For ferrocene the eclipsed $\mathrm{D}_{5 \mathrm{~h}}$ structure was found as global minimum at 


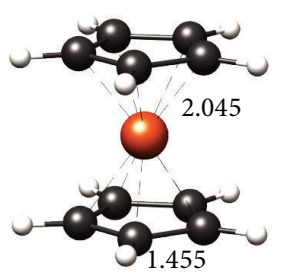

3

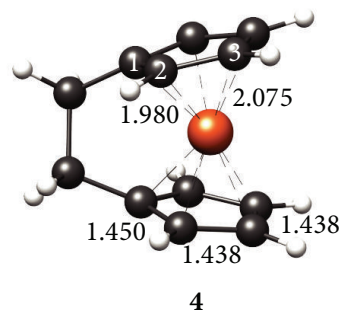

4
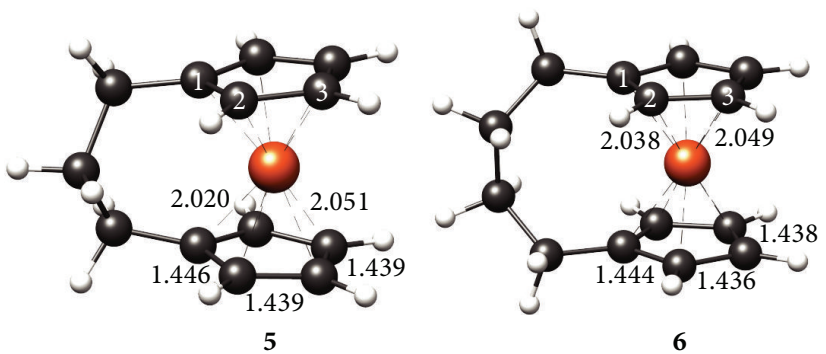

6

FIGURE 2: Computed structures of complexes 3 through 6. Bond lengths are given in $\AA$.

the PBEPBE level, in accord with the studies of Salzner [21]. In all ansa-complexes, the presence of the bridge breaks the symmetry of the $\mathrm{Cp}$ ring leading to contracted $\mathrm{C}-\mathrm{C}$ bonds in comparison to those in ferrocene. The $\mathrm{C} 1-\mathrm{C} 2$ bonds, in the neighboring position with respect to the bridge, are the least elongated, while the $\mathrm{C} 2-\mathrm{C} 3$ and $\mathrm{C} 3-\mathrm{C} 3^{\prime}$ bonds are somewhat shorter. According to bond lengths, the same tendency can be observed for the cavitands $\mathbf{1}$ and $\mathbf{2}$. As expected, the lengths of $\mathrm{Fe}-\mathrm{C}$ bonds strongly depend on the bridge, resulting in very short $\mathrm{Fe}-\mathrm{Cl}$ distance in complex 4 . In complex 6 , however, with the more flexible 1,4-butylene group, the $\mathrm{Fe}-\mathrm{C}$ distances are quite close to those in ferrocene. It is remarkable that the $\mathrm{Fe}-\mathrm{C}$ distances in cavitands are somewhat longer compared to ansa-ferrocenes. For instance, in complex 1 , the $\mathrm{Fe}-\mathrm{C} 3$ distance is $2.156 \AA$, notably longer than that in complex $\mathbf{4}$, with somewhat comparable tilt angle (see Table 1).

In Table 1, the results of natural population analysis have been collected as well. The NPA charges of iron centers show distinct values for cavitands and ansa-ferrocenes. In complexes $\mathbf{1}$ and $\mathbf{2}$, the iron atoms are more positive than those in ferrocene. In contrast, in complexes 4-6 the metal center is more negative. As expected, the charge distribution in the $\mathrm{Cp}$ rings strongly depends on the relative position of the bridge; the partial charges increase gradually from the ipsocarbons to the $\mathrm{C} 3$ carbons. The electron density distribution of the $\mathrm{Cp}$ rings in cavitands reveals similarity with that of complexes 4-6, especially for $\mathbf{2}$, which presumably possesses a less rigid structure compared to complex $\mathbf{1}$.

The electronic structure around the iron central atom has been elucidated within the framework of the quantum theory of atoms in molecules developed by Bader. One of the three QTAIM descriptors taken into account is the electron density at bond critical points $\left(\rho_{\mathrm{BCP}}\right)$, which is somewhat related bond strengths. The delocalization index $\delta(\mathrm{A}, \mathrm{B})$, which is introduced by Bader and Stephens [22], describes the number of electron pairs delocalized between two atomic basins. The $\delta(\mathrm{A}, \mathrm{B})$ is somewhat related to formal bond orders for an equally shared pair between two atoms in a polyatomic molecule; however, it is usually less than that due to delocalization over the other atoms in the molecule. The ellipticity $(\varepsilon)$ calculated from two negative eigenvalues $\left(\lambda_{1}\right.$ and $\left.\lambda_{2}\right)$ of the Hessian matrix of the electron density function $\rho(\mathbf{r})$ at the BCP is a measure of the deviation of the charge density from the axial symmetry of a chemical bond and is defined as $\varepsilon=\lambda_{1} / \lambda_{2}-1$. Values close to zero indicate cylindrical character, whereas values greater than
TABLE 1: Structural parameters (in degrees, see Scheme 1) and selected NPA charges of complexes 1-6.

\begin{tabular}{lcccccc}
\hline Complex & $\alpha$ & $\beta$ & $Q(\mathrm{Fe})$ & $Q(\mathrm{C} 1)$ & $Q(\mathrm{C} 2)$ & $Q(\mathrm{C} 3)$ \\
\hline $\mathbf{1}$ & -14.1 & -9.7 & 0.178 & -0.072 & -0.259 & -0.263 \\
$\mathbf{2}$ & -6.6 & -4.6 & 0.176 & -0.080 & -0.270 & -0.271 \\
$\mathbf{3}$ & 0 & n.a. & 0.141 & -0.281 & n.a. & n.a. \\
$\mathbf{4}$ & 21.0 & 14.8 & 0.124 & -0.043 & -0.261 & -0.297 \\
$\mathbf{5}$ & 8.4 & 4.4 & 0.120 & -0.061 & -0.269 & -0.283 \\
$\mathbf{6}$ & 1.8 & -2.4 & 0.128 & -0.065 & -0.273 & -0.277 \\
\hline
\end{tabular}

zero may indicate partial $\pi$-character of a bond. The $\rho_{\mathrm{BCP}}$ and $\varepsilon$ values for complexes 1-6 are compiled in Table 2, whereas delocalization indices $\delta(\mathrm{A}, \mathrm{B})$ are presented in Table 3 .

The introduction of a bridge causes changes in $\mathrm{Fe}-\mathrm{C}$ bond strengths due to the distortion of the metallocene structure. For the cavitand-based complexes, the interaction between the iron center and the $\mathrm{Cp}$ rings is somewhat weaker in comparison to ferrocene. In ansa-metallocenes, however, the strength of this interaction strongly depends on the bridge size: in the more strained complex 4 , the $\mathrm{Fe}-\mathrm{Cl}$ and $\mathrm{Fe}-$ $\mathrm{C} 2$ bonds become stronger, while the $\mathrm{Fe}-\mathrm{C} 3$ bond is weaker in terms of delocalization indices, as well as $\rho_{\mathrm{BCP}}$ values. As expected, the smallest change in the strength of $\mathrm{Fe}-\mathrm{C}$ interactions can be observed for the least strained ansaferrocene 6 .

Inspecting, however, the changes in electron density distribution of the $\mathrm{Cp}$ rings, it can be observed that the $\mathrm{C}-\mathrm{C}$ bond strengths, in terms of delocalization indices, are expected to be increased upon the introduction of the ansabridge for complexes $\mathbf{1}$ and $\mathbf{5}$ in $\beta$-position, whereas almost no change is predicted for complexes $\mathbf{2}, \mathbf{4}$, and $\mathbf{6}$. For the $\mathrm{Cl}-$ C2 bonds, a decrease can be expected in all cases according to the $\rho_{\mathrm{BCP}}$ and $\delta(\mathrm{A}, \mathrm{B})$ values. The bond ellipticities follow the distortion of the metallocene structure as well. Upon decreasing the distance between the analogous carbon atoms in the $\mathrm{Cp}$ rings, the double bond character is increased, whereas it is decreased for the carbon atoms on the opening parts in the rings. That is, in complexes $\mathbf{1}$ and $\mathbf{2}$, the $\mathrm{C} 2-\mathrm{C} 3$ bond is expected to behave with a somewhat increased double bond character, which may result in a change of the reactivity of the rings on that site.

It is concluded that the synthesis of ferrocene-substituted tetrakis(methyl)resorcin[4] arene (1) and tetrakis(phenoxymethyl)resorcin[4]arene (2) is likely to lead to success. 
TABLE 2: Electron densities and bond ellipticities in bond critical points $\rho_{\mathrm{BCP}}$ for selected bonds in complexes 1-6.

\begin{tabular}{lccccccc}
\hline Complex & $\rho_{\mathrm{BCP}}(\mathrm{Fe}, \mathrm{C} 1)$ & $\rho_{\mathrm{BCP}}(\mathrm{Fe}, \mathrm{C} 2)$ & $\rho_{\mathrm{BCP}}(\mathrm{Fe}, \mathrm{C} 3)$ & $\rho_{\mathrm{BCP}}(\mathrm{C} 1, \mathrm{C} 2)$ & $\varepsilon(\mathrm{C} 1, \mathrm{C} 2)$ & $\rho_{\mathrm{BCP}}(\mathrm{C} 2, \mathrm{C} 3)$ & $\varepsilon(\mathrm{C} 2, \mathrm{C} 3)$ \\
\hline $\mathbf{1}$ & 0.080 & 0.083 & 0.091 & 0.279 & 0.220 & 0.282 & 0.235 \\
$\mathbf{2}$ & 0.081 & 0.089 & 0.090 & 0.277 & 0.221 & 0.282 \\
$\mathbf{3}$ & 0.089 & n.a. & n.a. & 0.281 & 0.244 & 0.245 \\
$\mathbf{4}$ & 0.103 & 0.094 & 0.086 & 0.276 & 0.264 & n.a. & 0.279 \\
$\mathbf{5}$ & 0.094 & 0.091 & 0.088 & 0.278 & 0.253 & 0.282 & 0.243 \\
$\mathbf{6}$ & 0.090 & 0.090 & 0.088 & 0.279 & 0.239 & 0.281 & 0.244 \\
\hline
\end{tabular}

TABLE 3: Delocalization indices $\delta(\mathrm{A}, \mathrm{B})$ in complexes $\mathbf{1 - 6}$.

\begin{tabular}{|c|c|c|c|c|c|}
\hline Complex & $\delta(\mathrm{Fe}, \mathrm{C} 1)$ & $\delta(\mathrm{Fe}, \mathrm{C} 2)$ & $\delta(\mathrm{Fe}, \mathrm{C} 3)$ & $\delta(\mathrm{C} 1, \mathrm{C} 2)$ & $\delta(\mathrm{C} 2, \mathrm{C} 3)$ \\
\hline 1 & 0.467 & 0.501 & 0.503 & 1.189 & 1.223 \\
\hline 2 & 0.437 & 0.501 & 0.502 & 1.171 & 1.211 \\
\hline 3 & 0.503 & n.a. & n.a. & 1.211 & n.a. \\
\hline 4 & 0.517 & 0.510 & 0.491 & 1.172 & 1.210 \\
\hline 5 & 0.492 & 0.508 & 0.497 & 1.178 & 1.215 \\
\hline 6 & 0.491 & 0.505 & 0.501 & 1.182 & 1.211 \\
\hline
\end{tabular}

These complexes, with presumably somewhat activated ferrocene moieties, are possible candidates for further transformations. The increased electron density on the $\beta$-position of cyclopentadienyl rings makes them possible candidates for following functionalization.

\section{Conflict of Interests}

The authors declare that there is no conflict of interests regarding the publication of this paper.

\section{References}

[1] T. J. Kealy and P. L. Pauson, "A new type of organo-iron compound," Nature, vol. 168, no. 4285, pp. 1039-1040, 1951.

[2] P. J. Shapiro, "The evolution of the ansa-bridge and its effect on the scope of metallocene chemistry," Coordination Chemistry Reviews, vol. 231, no. 1-2, pp. 67-81, 2002.

[3] J. A. Smith, J. von Seyerl, G. Huttner, and H. H. Brintzinger, "ansa-Metallocene derivatives. Molecular structure and proton magnetic resonance spectra or methylene- and ethylenebridged dicyclopentadienyltitanium compounds," Journal of Organometallic Chemistry, vol. 173, no. 2, pp. 175-185, 1979.

[4] W. Kaminsky, K. Külper, H. H. Brintzinger, and F. R. W. P. Wild, "Polymerization of propene and butene with a chiral zirconocene and methylalumoxane as cocatalyst," Angewandte Chemie International Edition in English, vol. 204, no. 6, pp. 507508, 1985.

[5] H. L. Lentzner and W. E. Watts, "Bridged ferrocenes-VII. The preparation and properties of [2]ferrocenophanes," Tetrahedron, vol. 27, no. 18, pp. 4343-4351, 1971.

[6] G. Wilkinson, M. Rosenblum, M. C. Whiting, and R. B. Woodward, "The structure of iron bis-cyclopentadienyl," Journal of the American Chemical Society, vol. 74, no. 8, pp. 2125-2126, 1952.

[7] H. Schwemlein, L. Zsolnai, G. Huttner, and H. H. Brintzinger, "ansa-metallocene derivatives. VI. Synthesis and molecular structure of a stable tetramethylethylene-bridged chromocene carbonyl complex, $\left(\mathrm{CH}_{3}\right)_{4} \mathrm{C}_{2}\left(\mathrm{C}_{5} \mathrm{H}_{4}\right)_{2} \mathrm{Cr}(\mathrm{CO})$," Journal of Organometallic Chemistry, vol. 256, no. 2, pp. 285-289, 1983.
[8] K. L. T. Wong and H. H. Brintzinger, "Reactivity patterns of chromocene, molybdenocene, and tungstenocene reaction systems. I. Carbonyl complex formation as a probe of coordinative unsaturation," Journal of the American Chemical Society, vol. 97, no. 18, pp. 5143-5146, 1975.

[9] F. Schaper, M. Rentzsch, M.-H. Prosenc, U. Rief, K. Schmidt, and H.-H. Brintzinger, "ansa-Metallocene derivatives XXXVI. Dimethylsilyl-bridged permethyl chromocene carbonyl complexes-syntheses, crystal structures and interconversion reactions," Journal of Organometallic Chemistry, vol. 534, no. 1-2, pp. 67-79, 1997.

[10] G. J. Matare, D. M. Foo, K. M. Kane et al., "ansa-Chromocene complexes. 1. Synthesis and characterization of Cr(II) carbonyl and tert-butyl isocyanide complexes," Organometallics, vol. 19, no. 8, pp. 1534-1539, 2000.

[11] J. C. Green and C. N. Jardine, "Thermal stability of Group 6 bis(cyclopentadienyl) and ethylene bridged bis(cyclopentadienyl) monocarbonyl complexes; a theoretical study," Journal of the Chemical Society, Dalton Transactions, pp. 3767-3770, 1999.

[12] Z. Csók, T. Kégl, Y. Li et al., "Synthesis of elongated cavitands via click reactions and their use as chemosensors," Tetrahedron, vol. 69, no. 38, pp. 8186-8190, 2013.

[13] D. E. Korshin, N. V. Nastapova, S. V. Kharlamov et al., "Electroswitchable self-assembly of tetraferrocene-resorcinarene," Mendeleev Communications, vol. 23, no. 2, pp. 71-73, 2013.

[14] Y. Zhang, C. D. Kim, and P. Coppens, "Does C-methylcalix[4]resorcinarene always adopt the crown shape conformation? A resorcinarene/bipyridine/decamethylruthenocene supramolecular clathrate with a novel framework structure," Chemical Communications, pp. 2299-2300, 2000.

[15] J. P. Perdew, K. Burke, and M. Ernzerhof, "Generalized gradient approximation made simple," Physical Review Letters, vol. 77, no. 18, pp. 3865-3868, 1996.

[16] M. J. Frisch et al., Gaussian 09 Revision C. 01, Gaussian, Wallingford, Conn, USA, 2009.

[17] A. Schaefer, C. Huber, and R. Ahlrichs, "Fully optimized contracted Gaussian basis sets of triple zeta valence quality for atoms Li to Kr," Journal of Chemical Physics, vol. 100, p. 5829, 1994. 
[18] R. Ditchfield, W. J. Hehre, and J. A. Pople, "Self-consistent molecular-orbital methods. IX. An extended Gaussian-type basis for molecular-orbital studies of organic molecules," Journal of Chemical Physics, vol. 54, p. 724, 1971.

[19] NBO 5.0., E. D. Glendening, K. Badenhoop et al., Theoretical Chemistry Institute, University of Wisconsin, Madison, Wis, USA, 2001.

[20] AIMAll (Version 13. 05. 06), A. Todd, and T. K. Keith, Gristmill Software, Overland Park, Kan, USA, 2013.

[21] U. Salzner, "Quantitatively correct UV-vis spectrum of ferrocene with TDB3LYP," Journal of Chemical Theory and Computation, vol. 9, no. 9, pp. 4064-4073, 2013.

[22] R. F. W. Bader and M. E. Stephens, "Spatial localization of the electronic pair and number distributions in molecules," Journal of the American Chemical Society, vol. 97, no. 26, pp. 7391-7399, 1975. 

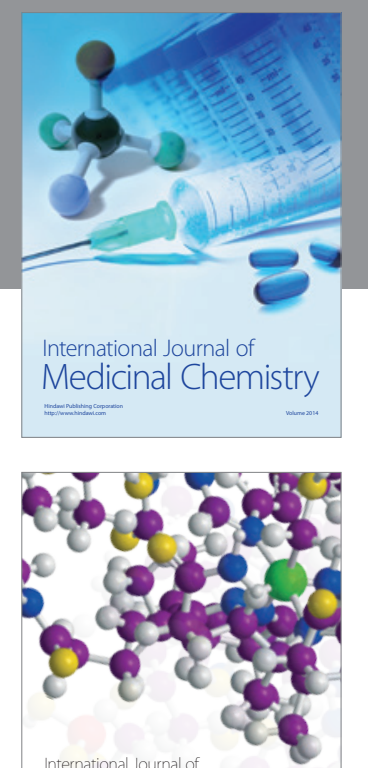

\section{Carbohydrate} Chemistry

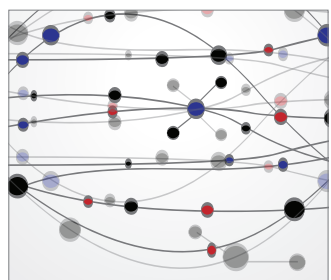

The Scientific World Journal
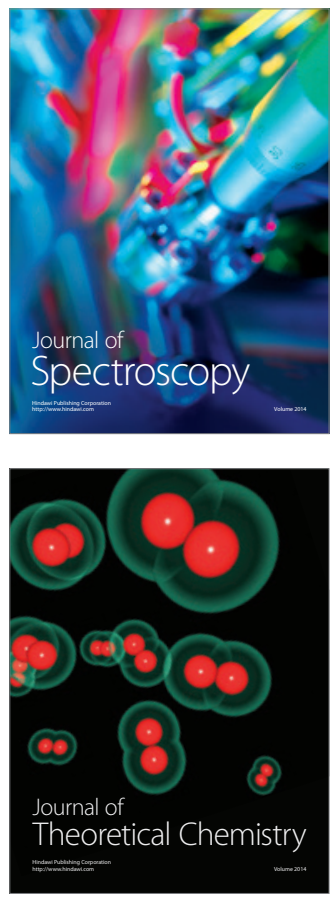
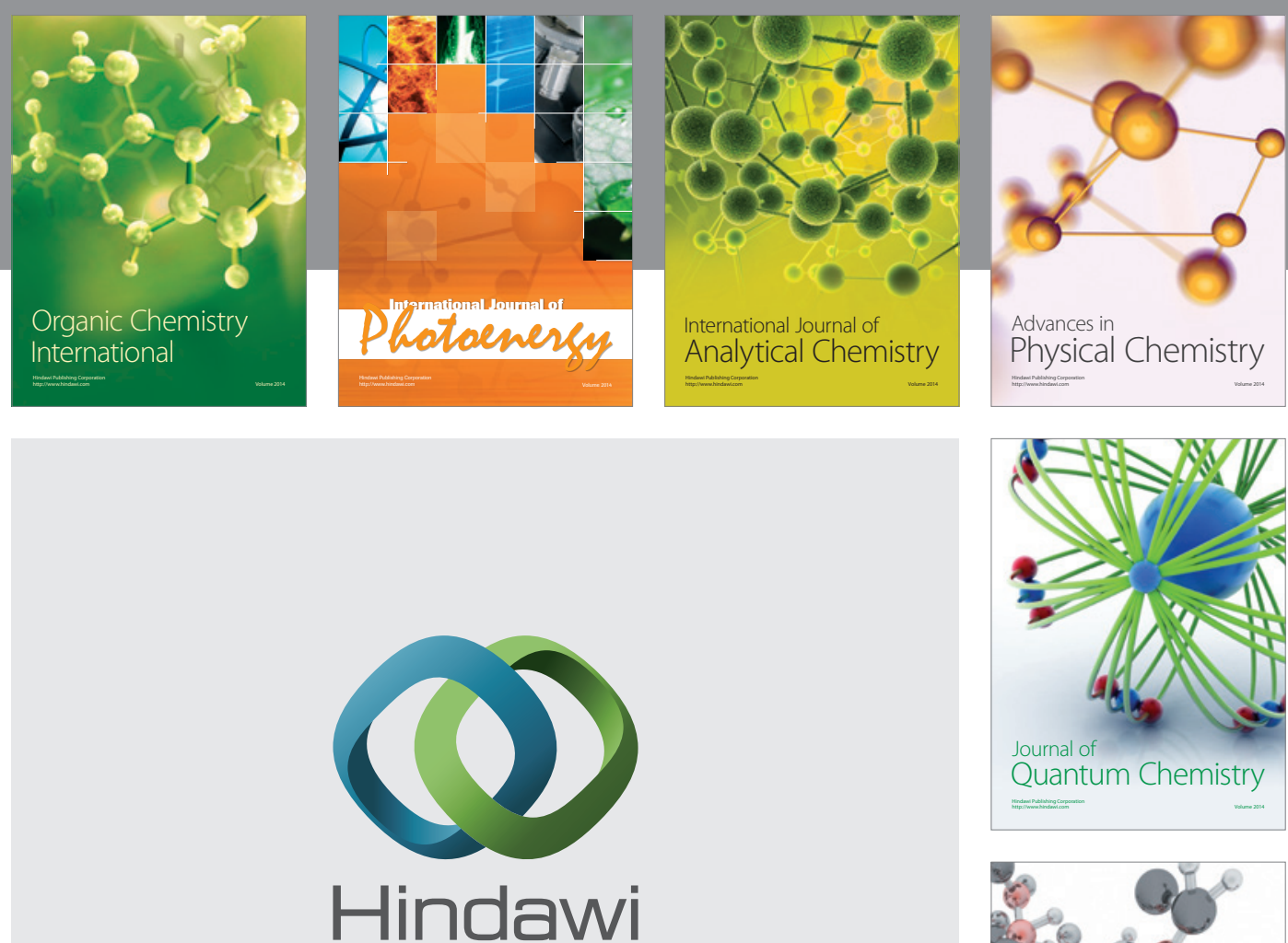

Submit your manuscripts at

http://www.hindawi.com

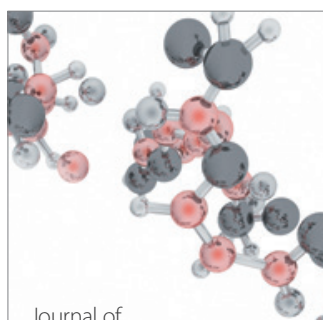

Analytical Methods

in Chemistry

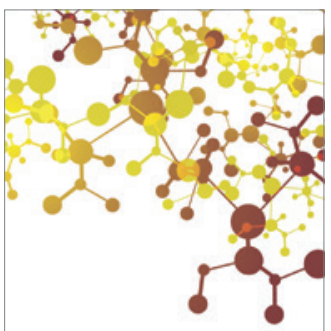

Journal of

Applied Chemistry

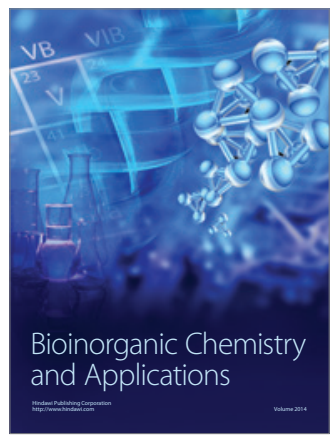

Inorganic Chemistry
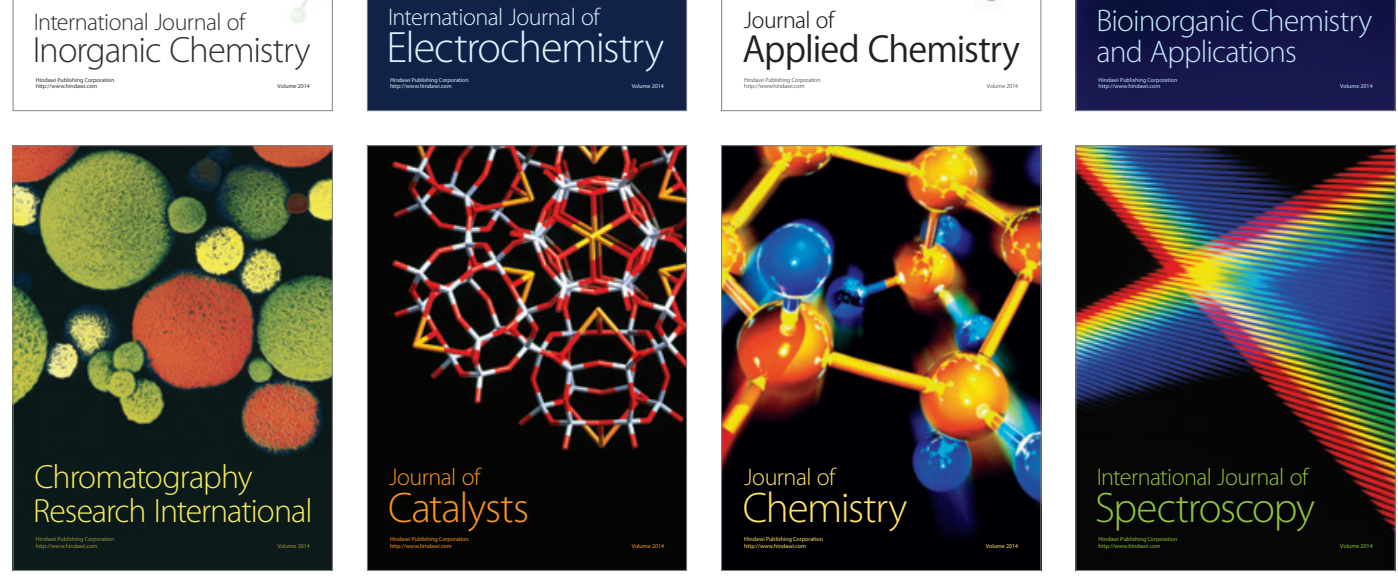